\title{
ON R-SECTORIAL DERIVATIVES ON BERGMAN SPACES
}

\author{
TOMÁŠ BÁRTA
}

(Received 15 August 2007)

\begin{abstract}
In this paper we show boundedness of vector-valued Bergman projections on simple connected domains. With this result we show R-sectoriality of the derivative on the Bergman space on $C_{+}$and maximal $L^{p}$-regularity for an integrodifferential equation with a kernel in the Bergman space.
\end{abstract}

2000 Mathematics subject classification: 47D06, 32A36.

Keywords and phrases: Bergman projection, R-sectoriality, integrodifferential equations, maximal regularity.

\section{Introduction}

The left shift semigroup on $\mathbb{R}_{+}$usually has very bad properties; it is not even normcontinuous. The spectrum of its generator, the derivative, contains the whole left halfplane. However, properties of the shift semigroup depend on the underlying function space. In [2] we introduced a left shift semigroup which is analytic, that is, its generator $d / d s$ is a sectorial operator. We have considered the shift semigroup on the space of bounded holomorphic functions on a sector with values in a Banach space $X$ equipped with the sup-norm, and the vector-valued Bergman space of $L^{p}$-integrable holomorphic functions on a sector, respectively. In the present paper we show that the generator $d / d s$ of the shift semigroup on the Bergman space is not just sectorial but also R-sectorial. This property corresponds to the maximal $L^{p}$-regularity for the transport equation

$$
\begin{gathered}
\dot{u}(t)=\frac{d}{d s} u(t)+f(t), \\
u(0)=u_{0} .
\end{gathered}
$$

The proof of R-sectoriality of $d / d s$ is based on the boundedness of the Bergman projection. So, in another main result of the present paper, we prove boundedness of the Bergman projection for vector-valued functions on various domains.

The main part of this paper was written during the author's stay at the University of Tübingen which was supported by the Marie Curie Fellowship. This work was further supported by GACR 201/06/P171 and MSM 113200007.

(C) 2008 Australian Mathematical Society 0004-9727/08 \$A2.00+0.00 
The original motivation for investigating analytic shift semigroups was the semigroup approach to the integrodifferential equation

$$
\begin{gathered}
\dot{u}(t)=A u(t)+\int_{0}^{t} B(t-s) A u(s) d s+f(t), \\
u(0)=x .
\end{gathered}
$$

In this approach (see for example [6]), the solution of (IDE) is described by the semigroup $\mathscr{T}$ generated by

$$
\mathscr{A}:=\left(\begin{array}{cc}
A & \delta_{0} \\
B(\cdot) & d / d s
\end{array}\right), \quad D(\mathscr{A}):=D(A) \times D(d / d s) .
$$

Hence, regularity of the solutions depends on regularity of the semigroup $\mathscr{T}$ and no regularity can be obtained by this approach if the shift semigroup generated by $d / d s$ is not even norm-continuous. In [2] we have shown that the solutions of (IDE) are analytic if the convolution kernel $B$ is analytic. In the present paper we show that the operator $\mathscr{A}$ is R-sectorial if $A$ is R-sectorial and $B$ belongs to an appropriate space of holomorphic functions. Hence, we have maximal $L^{p}$-regularity for (IDE). This last result is not very strong, since Weis showed in [11] maximal $L^{p}$-regularity for a class of kernels that are not necessarily holomorphic. However, the present result is not contained in Weis's result. Moreover, it gives a nice sufficient condition for maximal $L_{p}$-regularity, which is easy to verify.

This paper is organized as follows. In Section 2 we prove boundedness of the Bergman projection for vector-valued Bergman spaces. Section 3 deals with Rsectoriality of $d / d s$ and $\mathscr{A}$ and maximal $L^{p}$-regularity for (IDE). The main results are contained in Theorems 2.1,3.1, and 3.3.

\section{Boundedness of the Bergman projection}

The Bergman space $B^{q}(\Omega, X), \Omega \subset \mathbb{C}$, is defined by

$$
B^{q}(\Omega, X):=\left\{f: \Omega \rightarrow X: f \text { holomorphic and }\|f\|_{q}<+\infty\right\},
$$

where

$$
\|f\|_{q}^{q}:=\int_{\Omega}\|f(x+i y)\|^{q} d x d y .
$$

In this section we study the Bergman projection $\Pi$ of $L^{q}(\Omega, X)$ onto its closed subspace $B^{q}(\Omega, X)$. We show that the Bergman projection is a bounded operator on $L^{q}$ for all $q \in(4 / 3,4)$. Here $\Omega$ is a simply connected domain and $X$ is a Banach space. Moreover, we show that the projection is bounded for arbitrary $1<q<\infty$ if $\Omega$ is a sector

$$
\Sigma_{\theta}:=\{z \in \mathbb{C}: z \neq 0 \text { and }|\arg z|<\theta\}, \quad 0<\theta \leq \pi / 2
$$


Hedenmalm proved in [7] that the Bergman projection is bounded for scalar-valued functions, simply connected domains and $p \in(4 / 3,4)$. We use the idea of his proof. Concerning vector-valued functions, the boundedness of the Bergman projection is known for the unit disk and all $1<p<\infty$ (see Arregui and Blasco [1]).

Let $\psi$ be a conformal mapping from $\Omega$ onto the unit disk $D$. The Bergman projection for scalar-valued functions on $D$ is given by

$$
\Pi_{D} f(z):=\int_{D} K_{D}(z, w) f(w) d \lambda_{2}(w), \quad K_{D}(z, w):=\frac{1}{(1-z \bar{w})^{2}},
$$

and for functions on $\Omega$ by

$$
\Pi_{\Omega} f(u):=\int_{\Omega} K_{\Omega}(u, v) f(v) d \lambda_{2}(v), \quad K_{\Omega}(u, v):=\frac{\psi^{\prime}(u) \bar{\psi}^{\prime}(v)}{(1-\psi(u) \bar{\psi}(v))^{2}}
$$

(see Hedenmalm [7]). We define the Bergman projection for vector-valued functions by the same formula. We first show that $\Pi_{\Omega}$ is a projection onto $B^{q}(\Omega, X)$. In fact,

$$
x^{\prime}\left(\Pi_{\Omega} f(z)\right)=\Pi_{\Omega}\left(x^{\prime} \circ f\right)(z),
$$

for all $x^{\prime} \in X^{\prime}$. The right-hand side is a holomorphic function, since $\Pi_{\Omega}$ is a projection onto $B^{q}(\Omega, \mathbb{C})$ in the scalar case. Hence, $\Pi_{\Omega} f(\cdot)$ is holomorphic since the left-hand side is holomorphic for every $x^{\prime} \in X^{\prime}$. Moreover, if $f$ is holomorphic, then $x^{\prime} \circ f$ is holomorphic and (2.2) equals $\left(x^{\prime} \circ f\right)(z)$. Hence, $\Pi_{\Omega} f(z)=f(z)$.

We now show that the projection is bounded. In fact, we show that if we replace the kernel of the projection by its absolute value, then the mapping remains bounded. We shall need this stronger result in the proof of R-sectoriality of $d / d s$.

THEOREM 2.1. Let $q \in(4 / 3,4)$ and $\Omega$ be simply connected. Then the Bergman projection $\Pi_{\Omega}$ and the mapping $\Pi_{\Omega}^{*}$ defined by

$$
\left(\Pi_{\Omega}^{*} f\right)(u):=\int_{\Omega}\left|K_{\Omega}(u, v)\right| f(v) d v
$$

are bounded operators on $L^{q}(\Omega, X)$.

PROOF. It is sufficient to show boundedness of $\Pi_{\Omega}^{*}$, that is, the estimate

$$
\begin{gathered}
\int_{\Omega}\left\|\Pi_{\Omega}^{*}\left(\left|\psi^{\prime}\right| \cdot g\right)(u)\right\|^{q} d \lambda_{2}(u) \leq C \int_{\Omega}\left\|\left|\psi^{\prime}(u)\right| g(u)\right\|^{q} d \lambda_{2}(u), \\
\left|\psi^{\prime}\right| \cdot g \in L^{q}(\Omega, X) .
\end{gathered}
$$

The substitution $f(z):=g(\varphi(z))$, where $\varphi:=\psi^{-1}$, yields equivalence of the previous estimate and

$$
\begin{aligned}
& \int_{D}\left\|\varphi^{\prime}(z) \Pi_{\Omega}^{*}\left(\left|\psi^{\prime}\right| \cdot f \circ \psi\right) \circ \varphi(z)\right\|^{q}\left|\varphi^{\prime}(z)\right|^{2-q} d \lambda_{2}(z) \\
& \quad \leq \int_{D}\|f(z)\|^{q}\left|\varphi^{\prime}(z)\right|^{2-q} d \lambda_{2}(z) .
\end{aligned}
$$


We show that $\Pi_{D}^{*} f(z)=\left|\varphi^{\prime}(z)\right| \cdot \Pi_{\Omega}^{*}\left(\left|\psi^{\prime}\right| \cdot f \circ \psi\right) \circ \varphi(z), z \in D$, where

$$
\Pi_{D}^{*} f:=\int_{D}\left|\frac{1}{(1-z \bar{w})^{2}}\right| f(w) d \lambda_{2}(w) .
$$

In fact,

$$
\begin{aligned}
& \left|\varphi^{\prime}(z)\right| \cdot \Pi_{\Omega}^{*}\left(\left|\psi^{\prime}\right| \cdot f \circ \psi\right) \circ \varphi(z) \\
& \quad=\left|\varphi^{\prime}(z)\right| \int_{\Omega}\left|\frac{\psi^{\prime}(\varphi(z)) \bar{\psi}^{\prime}(v)}{(1-\psi(\varphi(z)) \bar{\psi}(v))^{2}}\right|\left|\psi^{\prime}(v)\right| \cdot f(\psi(v)) d v \\
& \quad=\int_{\Omega}\left|\frac{1}{(1-z \bar{\psi}(v))^{2}}\right|\left|\psi^{\prime}(v)\right|^{2} \cdot f(\psi(v)) d v=\int_{D}\left|\frac{1}{(1-z \bar{w})^{2}}\right| f(w) d w .
\end{aligned}
$$

So, it remains to prove

$$
\int_{D}\left\|\Pi_{D}^{*} f(z)\right\|^{q}\left|\varphi^{\prime}(z)\right|^{2-q} d \lambda_{2}(z) \leq \int_{D}\|f(z)\|^{q}\left|\varphi^{\prime}(z)\right|^{2-q} d \lambda_{2}(z) .
$$

This means that $\Pi_{D}^{*}$ is a bounded operator on the weighted space $L^{q}(D, X, \omega)$, with weight $\omega=\left|\varphi^{\prime}(z)\right|^{2-q}$.

We have the estimate

$$
\begin{aligned}
\left\|\Pi_{D}^{*} f(z)\right\| & =\left\|\int_{D}\left|K_{D}(z, w)\right| f(w) d \lambda_{2}(w)\right\| \\
& \leq \int_{D}\left|K_{D}(z, w)\right|\|f(w)\| d \lambda_{2}(w)=\Pi_{D}^{*}\|f(z)\| .
\end{aligned}
$$

Hence, it is sufficient to show that $\Pi_{D}^{*}$ is a bounded operator on $L^{q}(D, \mathbb{C}, \omega)$, that is, for scalar-valued functions $z \mapsto\|f(z)\|$. It was proved by Bekollé and Bonami in [3, Propositions 3 and 4] that $\Pi_{D}^{*}$ is a bounded operator on the weighted space $L^{q}(D, \mathbb{C}, \omega)$ if $\omega$ satisfies

$$
\sup _{(\theta, \rho) \in M}\left(\frac{1}{|S(\theta, \rho)|} \int_{S(\theta, \rho)} \omega d \lambda_{2}\right)\left(\frac{1}{|S(\theta, \rho)|} \int_{S(\theta, \rho)} \omega^{-1 /(q-1)} d \lambda_{2}\right)^{q-1} \leq C,
$$

with $M:=[0,2 \pi) \times(0,1]$. The set $S(\theta, \rho)$ is defined by

$$
S(\theta, \rho):=\left\{z=r e^{i \alpha}: 1-\rho<r<1,|\alpha-\theta|<2 \pi \rho\right\} .
$$

In Hedenmalm [7] it is shown that $\omega=\left|\varphi^{\prime}\right|^{2-q}$ satisfies (2.3) for $4 / 3<q<4$.

Let us show the following result.

THEOREM 2.2. The Bergman projection $\Pi_{\Omega}$ and the operator $\Pi_{\Omega}^{*}$ are bounded on $L^{q}(\Omega, X)$ for every $1<q<\infty$, provided $\Omega=\Sigma_{\theta}, 0<\theta \leq \pi / 2$. 
Proof. Fix $q \in(1,+\infty)$ and $a \in[1,+\infty)$ such that $z \mapsto z^{a}$ maps $\Sigma_{\theta}$ onto $\mathbb{C}_{+}$. Then

$$
\psi(u):=\frac{1-u^{a}}{1+u^{a}}
$$

is the conformal mapping from $\Sigma_{\theta}$ onto the unit disk $D$. Its inverse is

$$
\varphi(z):=\left(\frac{1-z}{1+z}\right)^{1 / a}
$$

According to the proof of the previous theorem, it is sufficient to show that (2.3) holds for $\omega=\left|\varphi^{\prime}\right|^{2-q}$. First of all, we show that the integrals

$$
\int_{D} \omega d \lambda_{2} \text { and } \int_{D} \omega^{-1 /(q-1)} d \lambda_{2}
$$

are finite. Since

$$
\left|\varphi^{\prime}(z)\right|=\frac{2}{a}|1+z|^{-1-1 / a}|1-z|^{-1+1 / a},
$$

we have

$$
c|1-z|^{(-1+(1 / a))(2-q)} \leq \omega(z) \leq C|1-z|^{(-1+(1 / a))(2-q),}
$$

for $z$ in a neighbourhood of 1 , and

$$
c|1+z|^{(-1-(1 / a))(2-q)} \leq \omega(z) \leq C|1+z|^{(-1-(1 / a))(2-q),}
$$

for $z$ in a neighbourhood of -1 , which are the only problematic points. Since the exponents are larger than -2 the first integral in (2.5) is finite. The proof for the second integral is analogous since

$$
\left(-1+\frac{1}{a}\right) \frac{2-q}{1-q}>-2 \text { and }\left(-1-\frac{1}{a}\right) \frac{2-q}{1-q}>-2,
$$

for $a \in[1, \infty), q \in(1, \infty)$.

Take $\varepsilon>0$ small enough. For $\rho>\varepsilon$ the integrals in (2.3) are estimated by the integrals over $D$ and $|S(\theta, \rho)| \geq \delta$ for some $\delta>0$. Hence,

$$
\left(\frac{1}{|S(\theta, \rho)|} \int_{S(\theta, \rho)} \omega d \lambda_{2}\right)\left(\frac{1}{|S(\theta, \rho)|} \int_{S(\theta, \rho)} \omega^{-1 /(q-1)} d \lambda_{2}\right)^{q-1}
$$

is estimated by a constant independent of $\theta, \rho$, provided $\rho>\varepsilon$.

It remains to show that (2.6) is bounded for $\rho \leq \varepsilon$. If $\varepsilon$ is small enough, then $S(\theta, \rho)$ is far enough from at least one of the points $1,-1$. The two cases are analogous, so we may assume that $\operatorname{dist}(-1, S(\theta, \rho)) \geq 5 \varepsilon$. Take $c>0$ such that

$$
S(\theta, \rho) \subset B\left(e^{i \theta}, c \rho\right), \quad \forall \theta \in[0,2 \pi), \rho \leq \varepsilon,
$$

where $B(x, r)$ denotes the ball of radius $r$ centred at $x$. 
Assume first that $d:=\operatorname{dist}\left(1, B\left(e^{i \theta}, c \rho\right)\right) \geq c \rho$. Then

$$
\int_{S(\theta, \rho)} C|1-z|^{\alpha} d \lambda_{2} \leq \int_{B\left(e^{i \theta}, c \rho\right)} C|1-z|^{\alpha} d \lambda_{2} \leq 2 c \rho \int_{d}^{d+2 c \rho} C t^{\alpha} d t,
$$

if $\alpha<0$. If $\alpha>0$ we have to replace $t^{\alpha}$ by $\left(c_{1} t\right)^{\alpha}$ in the last term. In both cases

$$
\frac{1}{|S(\theta, \rho)|} \int_{S(\theta, \rho)} C|1-z|^{\alpha} d \lambda_{2} \leq C \frac{1}{\rho}\left((d+c \rho)^{\alpha+1}-d^{\alpha+1}\right),
$$

since the area of $S(\theta, \rho)$ is proportional to $\rho^{2}$. The right-hand side of (2.7) is estimated by $\widetilde{C} d^{\alpha}$. Hence, (2.6) is less than

$$
\widetilde{C}_{1} d^{\alpha} \cdot\left(\widetilde{C}_{2} d^{(\alpha / 1-q)}\right)^{q-1}=C_{3},
$$

where the constants are independent of $\rho$ and $\theta$.

If $d<c \rho$ then $B\left(e^{i \theta}, c \rho\right) \subset B(1,3 c \rho)$ and

$$
\frac{1}{|S(\theta, \rho)|} \int_{S(\theta, \rho)} C|1-z|^{\alpha} d \lambda_{2} \leq C \frac{1}{\rho^{2}} \int_{0}^{3 c \rho} 2 \pi t \cdot t^{\alpha} d t \leq \widetilde{C} \rho^{\alpha} .
$$

Hence, (2.6) is estimated by

$$
\widetilde{C} \rho^{\alpha}\left(\widetilde{C}_{1} \rho^{(\alpha / 1-q)}\right)^{q-1}=C_{3} .
$$

This last estimate completes the proof.

\section{R-sectoriality and maximal regularity}

In this section we show R-sectoriality of the derivative on the Bergman space $B^{q}\left(\mathbb{C}_{+}, X\right)$, where $X$ is a UMD space. With this result we show maximal $L^{p}$ regularity for (IDE). It follows immediately from the definitions below that if $\mathscr{A}$ has maximal $L^{p}$-regularity, then so also does (IDE).

Let $T \in(0, \infty]$. We say that operator $A$ (or (IDE)) has maximal $L^{p}$-regularity for $T$ if for every $f \in L^{p}([0, T), X)$ there exists a unique solution $u$ of

$$
\begin{gathered}
\dot{u}(t)=A u(t)+f(t), \quad 0 \leq t<T, \\
u(0)=0
\end{gathered}
$$

(or (IDE)), such that $\dot{u}, A u \in L^{p}([0, T), X)$ and

$$
\|\dot{u}\|_{p}+\|A u\|_{p} \leq C_{A}\|f\|_{p}
$$

holds with $C_{A}$ independent of $f$. 
A set $M \subset L(X)$ is said to be $R$-bounded if there exists a constant $C$ such that

$$
\left\|\sum_{i=1}^{n} r_{i}(\cdot) T_{i} x_{i}\right\|_{L^{q}((0,1), X)} \leq C\left\|\sum_{i=1}^{n} r_{i}(\cdot) x_{i}\right\|_{L^{q}((0,1), X)}
$$

holds for all $n \in \mathbb{N}, T_{1}, \ldots, T_{n} \in M, x_{1}, \ldots, x_{n} \in X$ and one/all $q \in(1,+\infty)$. The functions $r_{i}$ are Rademacher functions or another system of functions from $[0,1]$ to $\{-1,1\}$ satisfying

$$
\int_{0}^{1} r_{i}(t) r_{j}(t) d t=0 \quad \forall i \neq j
$$

The smallest $C$ satisfying (3.2) is called the $R$-bound of $M$. An operator

$$
A: D(A) \subset X \rightarrow X
$$

is said to be R-sectorial if $\Sigma_{\theta} \subset \rho(A)$ and the set

$$
\left\{\lambda R(\lambda, A) \mid \lambda \in \Sigma_{\theta}\right\}
$$

is R-bounded for some $\theta>\pi / 2$.

The following holds for each operator $B$ on a Banach space $X$. If $B$ has maximal $L^{p}$-regularity for one $p \in(1, \infty)$ and one $T \in(0, \infty)$, then it has maximal regularity for all $p \in(1, \infty)$ and all $T \in(0, \infty)$ (see Dore [5]). Moreover, if $X$ is a UMD space then $B$ has maximal $L^{p}$-regularity for $T=\infty$ if and only if it is R-sectorial. An operator $B$ has maximal $L^{p}$-regularity for $T<\infty$ if and only if $B+\omega$ is R-sectorial for some $\omega \in \mathbb{R}$ (see Weis [10]).

From now on, assume $X$ to be a UMD space. Then $B_{\theta}^{q}:=B^{q}\left(\Sigma_{\theta}, X\right)$ is a UMD space, since it is a closed subspace of a UMD space $L^{q}\left(\Sigma_{\theta}, X\right)$. Hence, $\mathscr{X}^{q}=X \times B_{\theta}^{q}$ is a UMD space as well.

We show R-sectoriality of $d / d s$ in the case when $\theta=\pi / 2$. The proof of the following theorem is based on the idea of Weis.

Theorem 3.1. The operator $d / d$ s is $R$-sectorial on $B^{q}\left(\mathbb{C}_{+}, X\right), 1<q<+\infty$.

Proof. Since $f=\Pi f$ for $f \in B^{q}\left(\mathbb{C}_{+}, X\right)$, we have the following integral representation for the translation semigroup $T$ :

$$
(T(t) f)(z)=f(t+z)=\int_{\mathbb{C}_{+}} K(t+z, w) f(w) d w=\int_{\mathbb{C}_{+}} \frac{1}{(t+z+\bar{w})^{2}} f(w) d w .
$$

In fact, (2.1) and (2.4) yield

$$
K(u, v)=\frac{1}{(u+\bar{v})^{2}} .
$$

Since for all $t \in \Sigma_{\delta}, z, w \in \mathbb{C}_{+}$the inequality $|t+z+\bar{w}| \geq \frac{1}{2}|z+\bar{w}|$ holds (if $\delta$ is small enough), we obtain

$$
\left|\frac{1}{(t+z+\bar{w})^{2}}\right| \leq\left|\frac{4}{(z+\bar{w})^{2}}\right|
$$


Since the integral operator with kernel $k(z, w):=4|(z+\bar{w})|^{-2}$ is bounded on $L^{q}\left(\mathbb{C}_{+}, X\right)$ by Theorem 2.2, the set $\left\{T(t): t \in \Sigma_{\delta}\right\}$ is R-bounded by Denk et al. [4, Proposition 4.12]. Hence, the generator $d / d s$ is R-sectorial by Weis [10].

We shall now show that operator $\mathscr{A}$ defined by (1.1) is R-sectorial. To do this we apply perturbation theory for R-sectorial operators. We start with a perturbation lemma. It holds that $A+C$ is $\mathrm{R}$-sectorial if $A$ is $\mathrm{R}$-sectorial and $\|C x\| \leq a\|A x\|+$ $b\|x\|$ with $a$ sufficiently small. We show that if $C$ is of a special form then we do not need $a$ to be small. A similar result for sectorial operators was proved by Nagel (see [9]).

Lemma 3.2. Let $A: D(A) \rightarrow X \quad$ and $B: D(B) \rightarrow Y \quad$ be $R$-sectorial. If $C \in B(D(B), X)$, then

$$
E:=\left(\begin{array}{cc}
A & C \\
0 & B
\end{array}\right), \quad D(E):=D(A) \times D(B),
$$

is $R$-sectorial on $X \times Y$.

PROOF. The resolvent of $E$ is given by

$$
R(\lambda, E)=\left(\begin{array}{cc}
R(\lambda, A) & R(\lambda, A) C R(\lambda, B) \\
0 & R(\lambda, B)
\end{array}\right) .
$$

The set $\{\lambda R(\lambda, E): \lambda>0\}$ is R-bounded since $\{\lambda R(\lambda, A): \lambda>0\}$ and $\{\lambda R(\lambda, B)$ : $\lambda>0\}$ are R-bounded by assumption and the third term is R-bounded since for the R-bounds the inequality $\mathcal{R}(\lambda R(\lambda, A) C R(\lambda, B)) \leq \mathcal{R}(\lambda R(\lambda, A)) \cdot\left\|C(B+\varepsilon)^{-1}\right\| \cdot$ $\mathcal{R}((B+\varepsilon) R(\lambda, B))<+\infty$ holds.

TheOREM 3.3. Let A be R-sectorial on $X$ and $d / d$ s be R-sectorial on $B_{\theta}^{q}$ for some $q>2$. Let $B$ satisfy one of the following conditions:

(i) $B \in B^{q}\left(\Sigma_{\theta}, B(D(A), X)\right),\|B(\cdot) x\| \leq a\|A x\|+b\|x\|$ with $a>0$ sufficiently small;

(ii) $B \in B^{q}\left(\Sigma_{\theta}, B\left(X_{\alpha}, X\right)\right)$, where $X_{\alpha}=D\left(A^{\alpha}\right), 0<\alpha<1$;

(iii) $B, B^{\prime} \in B^{q}\left(\Sigma_{\theta}, B(D(A), X)\right)$.

Then $\mathscr{A}+w$ is $R$-sectorial for some $w \in \mathbb{R}$.

PROOF. According to the previous lemma,

$$
\mathscr{A}_{0}+w:=\left(\begin{array}{cc}
A+w & \delta_{0} \\
0 & d / d s+w
\end{array}\right)
$$

is R-sectorial for some $w \in \mathbb{R}$. Boundedness of $\delta_{0}: D(d / d s) \rightarrow D(A)$ follows from the continuous embedding of $W^{1, q}\left(\Sigma_{\theta}, X\right)$ in continuous functions for $q>2$. We can assume that $w=0$.

R-sectoriality of $\mathscr{A}+w$ now follows from [8, Theorem 1, if (i) holds, respectively Corollary 12, if (ii) holds]. If $B$ satisfies (iii), we consider the Banach space

$$
\mathscr{X}_{1}:=\left(D\left(\mathscr{A}_{0}\right),\|\cdot\|_{\mathscr{A}_{0}}\right),
$$


where $\|\cdot\|_{\mathscr{A}_{0}}$ denotes the graph norm. Since $\mathscr{X}_{1}$ is isomorphic to $\mathscr{X}$, it is also a UMD space. The part of $\mathscr{A}_{0}$ in $\mathscr{X}_{1}$ is similar to $\mathscr{A}_{0}$, and hence is R-sectorial. Since the part of $\mathscr{A}$ in $\mathscr{X}_{1}$ is a bounded perturbation of the part of $\mathscr{A}_{0}$ in $\mathscr{X}_{1}$, it is R-sectorial as well (see Kunstmann and Weis [8] or Dore [5]). By similarity we obtain the R-sectoriality of $\mathscr{A}$.

In this case, we have maximal $L^{p}$-regularity for the abstract Cauchy problem on the product space $\mathscr{X}$ on $[0, T)$ for every $T<\infty$. By taking the right-hand side $\mathscr{F}:=\left(\begin{array}{l}f \\ 0\end{array}\right)$, we obtain maximal $L^{p}$-regularity for (IDE). If $s(\mathscr{A})<\infty$, then we have maximal $L^{p}$-regularity for $T=\infty$ as well (see Dore [5]).

\section{Acknowledgements}

The author is grateful to R. Nagel for initiating his interest in this topic and L. Weis for help with R-boundedness.

\section{References}

[1] J. L. Arregui and O. Blasco, 'Bergman and Bloch spaces of vector-valued functions', Math. Nachr. 261 (2003), 3-22.

[2] T. Bárta, 'Analytic solutions of Volterra equations via semigroups', Semigroup Forum 76(1) (2008), 142-148.

[3] D. Bekollé and A. Bonami, 'Inégalités à poids pour le noyau de Bergman', C. R. Acad. Sci. Paris Sér. A-B 286 (1978), A775-A778.

[4] R. Denk, M. Hieber and J. Prüss, 'R-boundedness, Fourier multipliers and problems of elliptic and parabolic type', Mem. Amer. Math. Soc. 166(788) (2003).

[5] G. Dore, 'Maximal regularity in $L^{p}$ spaces for an abstract Cauchy problem', Adv. Differential Equations 5 (2000), 293-322.

[6] K. J. Engel and R. Nagel, One-parameter semigroups for linear evolution equations, Graduate Texts in Mathematics, 194 (Springer, Berlin, 2000).

[7] H. Hedenmalm, 'The dual of a Bergman space on simply connected domains', J. Anal. Math. 88 (2002), 311-335.

[8] P. Kunstmann and L. Weis, 'Perturbation theorems for maximal $L_{p}$-regularity', Ann. Sc. Norm. Super. Pisa Cl. Sci. 30 (2001), 415-435.

[9] R. Nagel, 'Towards a 'matrix theory' for unbounded operator matrices', Math. Z. 201 (1989), $57-68$.

[10] L. Weis, 'Operator-valued Fourier multiplier theorems and maximal $L^{p}$-regularity', Math. Ann. 319 (2001), 735-758.

[11] L. Weis, A new approach to maximal $L_{p}$-regularity, evolution equations and their applications in physical and life sciences (Bad Herrenalb, 1998), Lecture Notes in Pure and Applied Mathematics, 215 (Marcel Dekker, New York, 2001), pp. 195-214.

TOMÁŠ BÁRTA, Department of Mathematical Analysis,

Faculty of Mathematics and Physics, Charles University, Prague, Sokolovska 83,

18000 Prague 8, Czech Republic

e-mail: barta@karlin.mff.cuni.cz 